\title{
MODALIDADES DE PODA AVALIADAS NA ARBORIZAÇÃO VIÁRIA SOB REDE ELÉTRICA NO ESTADO DE MINAS GERAIS
}

\author{
PRUNING TYPES EVALUATED FOR THE TREES THAT WERE LOCATED UNDER \\ POWER GRIDS IN THE STATE OF MINAS GERAIS-BRAZIL
}

\begin{abstract}
Altamir Fernandes de Oliveira ${ }^{1}$, José Aldo Alves Pereira ${ }^{2}$, Gabriel de Assis Pereira ${ }^{3}$, Silvério José Coelho ${ }^{4}$, Cinara Libéria Pereira Neves ${ }^{5}$, Samuel Wanderley Rezende ${ }^{6}$, Fernando Henrique Silva Garcia7
\end{abstract}

\begin{abstract}
RESUMO
O manejo de árvores em áreas urbanas é uma atividade complexa, em que os vários elementos da cidade devem ser levados em consideração. A compatibilidade das árvores com a rede elétrica é um dos grandes desafios dos gestores públicos e os serviços de poda são os mais onerosos na manutenção dos indivíduos arbóreos. Assim, o presente trabalho teve como objetivo, o diagnóstico das modalidades de podas empregadas no estado de Minas Gerais, bem como as causas dessa prática. Para isso, foram amostrados 80 circuitos elétricos em 35 cidades. Foram identificados 1.643 indivíduos arbóreos, classificados em 41 famílias e 130 espécies, sendo 54 exóticas (11 de pequeno porte, 14 de médio porte e 28 de grande porte) e 76 nativas (sete de pequeno porte, dez de médio porte e 58 de grande porte). Percebeu-se que mais da metade das árvores avaliadas $(52,28 \%)$ foram podadas por causa de conflitos com a fiação, sendo as espécies Licania tomentosa (Benth.) Fritsch (oiti), Ligustrum lucidum W.T. Aiton (alfeneiro) e Poincianella pluviosa (DC.) L.P.Queiroz (sibipiruna) as mais frequentes. A espécie introduzida sob rede elétrica também influenciou na modalidade da poda empregada, como por exemplo, a poda "em V" foi característica da Poincianella pluviosa.
\end{abstract}

Palavras-chave: Planejamento urbano; Distribuição de energia; Manejo integrado.

\section{ABSTRACT}

The trees management in urban areas is a complex activity and the various elements of the city must have been taken into account. The compatibility of the trees and the power grids is a major challenge for public managers and the pruning services are the most costly services in the maintenance of individual trees. Thus, this study aimed to diagnose the types of pruning employed in the State of Minas Gerais, as well as the causes of this practice. For this, there were sampled 80 electric circuits in 35 cities. The number of 1,643 recorded trees were classified into 41 families and 130 species, 54 exotic (11 small, 14 medium-sized and 28 large) and 76 native (seven small, ten medium-sized and 58 large. It was noticed that more than half of the assessed trees $(52.28 \%)$ were pruned because of conflicts with the wires, and the species Licania tomentosa (Benth.) Fritsch (oiti), Ligustrum lucidum W.T. Aiton (alfeneiro) and Poincianella pluviosa (DC.) L.P.Queiroz (sibipiruna) were the most frequent. The introduced species under power grids also influenced the mode of pruning employed, such as "V" pruning was characteristic of Poincianella pluviosa species.

Keywords: Urban planning; Energy distribution; Integrated management.

Recebido em 13.10.2015 e aceito em 11.11.2015

1 Biólogo, Administrador Público, Professor Doutor do Instituto de Ciência e Tecnologia da Universidade Federal dos Vales do Jequitinhonha e Mucuri - UFVJM-MG. Câmpus Mucuri, Rua do E-mail: altamirf3@gmail.com

2 Engenheiro Florestal, Professor Doutor do Departamento de Engenharia Florestal da Universidade Federal de Lavras UFLA-MG. Câmpus Universitário, Caixa Postal 3037. Lavras-MG. E-mail: j.aldo@dcf.ufla.br

3 Engenheiro Florestal, Mestre em Engenharia Florestal da UFLA-MG. E-mail: gabriel_assispereira@hotmail.com

4 Engenheiro Agrônomo, Professor Doutor do Departamento de Agronomia da UFLA-MG. E-mail: sjcoelho65@gmail.com.

5 Engenheira Agrônoma, Mestre em Fitotecnia pela UFLA-MG. E-mail: cinaraliberia@hotmail.com

6 Engenheiro Agrônomo, Mestre em Fitotecnia pela UFLA-MG. E-mail: samuelwr88@gmail.com

7 Engenheiro Agrônomo, Mestre em Fisiologia Vegetal pela UFLA-MG. E-mail: fernandohenriquesilva@hotmail.com 


\section{INTRODUÇÃO}

As árvores no meio urbano são elementos integrais que fazem parte do cotidiano das cidades, fornecendo benefícios ímpares nos recintos comerciais e residenciais, dentre eles ambientais, econômicos e sociais (MULLANEY; LUCKE; TRUEMAN, 2015).

O planejamento da arborização no meio urbano exige um processo cuidadoso que preveja os procedimentos desde sua concepção até sua implantação e manutenção (CEMIG, 2011). Para o sucesso em planos de arborização urbana o planejamento é de suma importância, pois se não for feito de maneira adequada pode gerar danos, problemas e prejuízos futuros (BOBROWSKI, 2011).

Assim, para a inserção de árvores nas cidades devem-se selecionar espécies mais adequadas ao espaço físico disponível, às condições ambientais e antrópicas locais. Em vias públicas, com a presença de rede elétrica, devem-se priorizar espécies de menor porte, ou que apresentam possibilidade de condução da copa e crescimento lento, para evitar conflitos futuros (CEMIG, 2011).

Para evitar e/ou remediar o conflito de espécies incompatíveis, são necessários trabalhos de podas e/ou remoção dos indivíduos, sendo justamente essas as atividades mais onerosas relacionadas à manutenção da arborização de ruas (NOWAK; MCBRIDE; BEATTY, 1990; CAMPANELLA; TOUSSAINT; PAUL, 2009; ROY; BYRNE; PICKERING, 2012).

Alguns tipos de poda não são recomendados, pois além de comprometer o estado fitossanitário da árvore, configuram crimes ambientais, como é o caso da destopa, uma prática que consiste na retirada da copa da árvore, muito comum no Brasil, tendo sua origem na colonização portuguesa. Segundo Campanella, Toussaint e Paul (2009), apesar de ser um método rápido e barato em curto prazo, a destopa causa efeitos deletérios às árvores, o que resulta na necessidade de poda mais frequente para sua correção, o que gera um aumento superior a quatro vezes o valor inicial, caso fosse feita a poda correta.

Para as concessionárias de energia elétrica, uma alternativa ao destopo é a poda direcional, com o objetivo de livrar a fiação elétrica dos galhos conflitantes. Essa modalidade de poda vem sendo utilizada nos Estados Unidos há mais de 30 anos (KUHNS; REITER, 2007), e consiste no corte de galhos indesejáveis que crescem em direção aos equipamentos ou instalações, e é mais eficaz quando são levados em consideração o tamanho, a forma e a taxa de crescimento da árvore (CEMIG, 2011). No Brasil, a adoção da poda direcional pelas concessionárias de energia elétrica é recente e pode ser percebida pelos tipos "em V" e unilateral. 
Árvores de pequeno porte e redes de distribuição de energia elétrica podem coexistir em uma situação que combine a estética e a ausência de riscos, caso os requisitos de tamanho e manutenção sejam considerados quando da escolha das espécies. $O$ uso de portes adequados reduz significativamente as interrupções elétricas assim como os custos de remoção e poda, e por isso deve ser avaliada a criação de arboretos ou viveiros por iniciativa das concessionárias de energia elétrica, ou por meio de parcerias com instituições afins (APPLETON, 2006).

Assim, objetivou-se com esse trabalho, realizar um diagnóstico das podas de árvores localizadas sob rede de distribuição de energia elétrica em 35 cidades do estado de Minas Gerais, com a finalidade de quantificá-las, para entender seus motivadores e sugerir alternativas para seu devido manejo.

\section{MATERIAL E MÉTODOS}

A coleta dos dados foi realizada em 35 cidades do Estado de Minas Gerais. Para isso levou-se em consideração a divisão do Estado em sete regionais administrativas adotada pela Companhia Energética de Minas Gerais (Cemig), são elas: Centro, Leste, Mantiqueira, Norte, Oeste, Sul e Triângulo.

Foram diagnosticadas cinco cidades por regional, a seleção considerou os relatórios tabelados das cidades de Minas Gerais que mais apresentaram desligamentos de energia elétrica relacionados ao conflito com árvores nos anos de 2011 e 2012, fornecidos em planilha Excel ${ }^{\circledR}$ pela Cemig.

Com base na metodologia descrita, as cidades selecionadas para cada regional foram: Regional Oeste (Arcos, Divinópolis, Formiga, Itaúna e Pará de Minas); Regional Sul (Alfenas, Guaxupé, Itajubá, Passa Quatro e Três Corações); Regional Centro (Betim, Contagem, Esmeraldas, Nova Lima e Ribeirão das Neves); Regional Mantiqueira (Conselheiro Lafaiete, Juiz de Fora, Itabirito, Moeda e Ouro Preto); Regional Leste (Araçuaí, Coronel Murta, Governador Valadares, Ipatinga e Timóteo); Regional Triângulo (Araguari, Ituiutaba, Patos de Minas, Uberaba e Uberlândia); Regional Norte (Curvelo, João Pinheiro, Montes Claros, Paracatu e Unaí).

Então, procurou-se identificar e localizar os circuitos, tidos como unidades amostrais com extensões variadas (extensão mínima de um quarteirão), para cada cidade. Esses circuitos fizeram parte do conjunto estratificado por regionais, analisado de acordo com todas as interrupções de energia elétrica causadas por árvores nos anos de 2011 e 2012. A melhor rota para os trabalhos de campo em cada cidade foi escolhida com o auxílio 
de técnicos da Cemig, os quais ajudaram na análise e localização dos circuitos a ser amostrados.

Inicialmente fez-se a análise visual de toda a extensão do circuito elétrico presente nas ruas e/ou avenidas, com o objetivo de verificar a quantidade de árvores a serem avaliadas. A amostragem foi tida como censo, pois considerou todos os indivíduos arbóreos localizados sob a rede (mudas e árvores). Quando não foram encontrados indivíduos arbóreos no circuito, selecionou-se o próximo com a presença de pelo menos um espécime. Os circuitos ausentes de árvores em seus trechos foram devido à eliminação das mesmas que estavam tocando a rede, causando desligamentos.

Definidos os circuitos, a serem diagnosticados, foram coletadas as informações da modalidade de poda realizada e suas causas. As modalidades avaliadas foram condução/formação, unilateral; destopa, "em V" ou inexistente e o motivo dessas foram sinalização/placas, semáforo, iluminação pública, edificação ou poste/rede. Posteriormente aos trabalhos de campo, compôs-se uma planilha Excel $\AA$ compilando todas as fichas das árvores avaliadas nas sete regionais, formando, assim, um banco de dados com os atributos coletados.

Para as espécies sem possibilidade de identificação imediata, arquivaram-se fotografias da árvore inteira, do tronco, das folhas, dos frutos e flores, quando possível. Esse conjunto de dados foi arquivado em meio lógico no Laboratório de Pesquisa em Arborização Urbana - LAPAR-Ufla e se encontra para consulta. Material para a montagem de exsicatas foi também coletado, com o objetivo de auxiliar na identificação das espécies por especialistas da Universidade Federal de Lavras (UFLA), da Universidade Federal de Minas Gerais (UFMG) e da Cemig. Foi necessário solicitar a esses especialistas a identificação de 68 espécies.

Para certificar a identificação, foi feita a conferência dos nomes científicos, com as sinonímias substituídas pelos nomes aceitos consultando banco de dados e bibliografias conforme protocolo de Cupertino e Eisenlohr (2013).

Posteriormente, identificou-se a origem das espécies, se nativas (do território brasileiro) ou exóticas, consultando-se bibliografias específicas, bem como a altura potencial, segundo Aguirre Júnior e Lima (2007), Araujo et al. (2012) e Melazo e Nishiyama (2010), que estratificam as espécies arbóreo urbanas em pequeno porte $(P$, menor ou igual a $6 \mathrm{~m}$ ), médio porte ( $M$, entre 6 e $10 \mathrm{~m}$ ) e grande porte $(\mathrm{G}$, maior que $10 \mathrm{~m})$, pois essas são classes que possuem melhor correlação com as alturas mínimas da fiação elétrica (CEMIG, 1996, 2002), para os diversos contextos na área urbana, nos municípios abrangidos pelo sistema elétrico da Cemig. 
Faz-se aqui a ressalva de que, não houve a intenção de adentrar na seara da discussão sobre a origem das espécies arbóreas, e, por mais que o Brasil seja demasiado grande para considerar se tal espécie é nativa do país como algo ecologicamente significativo, o ambiente urbano, com todas as suas complexidades, não é um ambiente florestal. Logo, a identificação da origem das espécies avaliadas nesse diagnóstico foi feita com a mera tentativa de complementar a pesquisa, bem como incrementar essa linha de pesquisa, ainda, em sua fase inicial no Brasil, e, mais ainda, incentivar novos estudos quanto às espécies atualmente utilizadas e a serem utilizadas no meio urbano.

Foi necessário realizar uma atualização dos nomes científicos, com as sinonímias sendo substituídas pelo nome mais aceito. Nesse caso, utilizou-se o banco de dados da Lista de Espécies da Flora do Brasil ( 2012) e o The Plant List (2013).

As análises de agrupamento para essas matrizes foram feitas no programa PAST 2.0, segundo Hammer et al. (2001), utilizando o algoritmo UPGMA (agrupamento de médias aritméticas não ponderadas).

Para a análise de correspondência canônica entre as espécies mais frequentes e os tipos de poda executados, prepararam-se matrizes de porcentagem ou de quantidade de indivíduos, incluindo as espécies nas linhas e os atributos (dimensões, variáveis) de tipos de poda executados nas colunas, os quais foram analisados no programa PAST 2.0, segundo Hammer et al. (2001).

\section{RESULTADOS E DISCUSSÃO}

Foram percorridos neste diagnóstico 80 circuitos ou trechos de redes elétricas, com a abrangência de 7.599 unidades consumidoras alimentadas em baixa e média tensão. Avaliaram-se 1.643 indivíduos, em que 69,08\% das árvores sofreram algum tipo de poda (1.135 árvores), as quais foram relativas a alguma modalidade ou combinação das podas de "condução ou formação", "unilateral", "destopa" ou "em V". Essas intervenções foram motivadas por moradores, executadas pela prefeitura ou pela concessionária de energia elétrica, com objetivos variados, tais como podas para livrar a fiação de galhos conflitantes, podas culturais municipais (incluindo destopa), podas estéticas, dentre outras.

Desse montante, as podas que foram motivadas apenas pelos conflitos com a rede elétrica, foram realizadas em 859 indivíduos, ou seja, 52,28\% das árvores avaliadas. Destas, 707 estavam relacionadas às 20 espécies mais frequentes $(75,59 \%$ do total dos indivíduos) identificadas no diagnóstico (43,03\%) (Tabela 1). 
Tabela 1 Quantidade de indivíduos relacionados às modalidades de podas executadas por causa da rede elétrica nas 20 espécies mais frequentes das 35 cidades avaliadas. Dados: $\mathrm{N}^{\circ}$ (Ranking das espécies identificadas), $\mathrm{N}$ (quantidade de indivíduos avaliados), Pr. (Porte: $\mathrm{P}=$ até $6 \mathrm{~m}, \mathrm{M}=$ de 6 a $10 \mathrm{~m}, \mathrm{G}=$ maior que $10 \mathrm{~m}$ ), FR (Frequência Relativa), CF (Poda de Condução ou Formação), U (Poda Unilateral), D (Destopa), V (Poda "em V")

Table 1 Number of trees related to the types of pruning performed because of the power grid in the 20 most common species of the 35 evaluated cities. Data: $\mathrm{N}^{\circ}$ (Species identified ranking), $\mathrm{N}$ (number of evaluated trees), Pr. (Size: $P=$ up to $6 \mathrm{~m}, M=6$ to $10 \mathrm{~m}, \mathrm{G}=$ greater than $10 \mathrm{~m}$ ), FR (Relative Frequency), CF (Forming Pruning or Conducting Pruning), U (Unilateral Pruning), D (Topping), V ("V" Pruning)

\begin{tabular}{|c|c|c|c|c|c|c|c|c|c|c|}
\hline $\mathbf{N}^{\circ}$ & Espécie & $\begin{array}{l}\text { Nome } \\
\text { comum }\end{array}$ & $\mathbf{N}$ & Pr. & FR & CF & $\mathbf{U}$ & D & v & Soma \\
\hline 1 & $\begin{array}{l}\text { Licania tomentosa } \\
\text { (Benth.) Fritsch }\end{array}$ & Oiti & 316 & $\mathrm{G}$ & $19,23 \%$ & 20 & 25 & 80 & 77 & 202 \\
\hline 2 & $\begin{array}{l}\text { Poincianella pluviosa } \\
\text { (DC.) L.P.Queiroz }\end{array}$ & Sibipiruna & 217 & $\mathrm{G}$ & $13,21 \%$ & 4 & 34 & 59 & 90 & 187 \\
\hline 3 & $\begin{array}{l}\text { Murraya paniculata (L.) } \\
\text { Jacq. }\end{array}$ & Murta & 98 & $P$ & $5,96 \%$ & 2 & 1 & 6 & 0 & 9 \\
\hline 4 & Ficus benjamina L. & $\begin{array}{l}\text { Fícus- } \\
\text { benjamina }\end{array}$ & 69 & $\mathrm{G}$ & $4,20 \%$ & 2 & 3 & 22 & 8 & 35 \\
\hline 5 & $\begin{array}{l}\text { Ligustrum lucidum W.T. } \\
\text { Aiton }\end{array}$ & Alfeneiro & 63 & M & $3,83 \%$ & 7 & 19 & 8 & 21 & 55 \\
\hline 6 & Hibiscus rosa-sinensis L. & Hibisco & 53 & $P$ & $3,23 \%$ & 10 & 0 & 0 & 0 & 10 \\
\hline 7 & $\begin{array}{l}\text { Lagerstroemia indica (L.) } \\
\text { Pers. }\end{array}$ & Resedá & 50 & $P$ & $3,04 \%$ & 2 & 0 & 5 & 0 & 7 \\
\hline 8 & Bauhinia variegata $\mathrm{L}$. & $\begin{array}{l}\text { Pata-de- } \\
\text { vaca }\end{array}$ & 47 & M & $2,86 \%$ & 3 & 4 & 17 & 8 & 32 \\
\hline 9 & $\begin{array}{l}\text { Callistemon viminalis } \\
\text { (Sol. Ex Gaertn.) G. Don } \\
\text { ex Loud. }\end{array}$ & Calistemo & 44 & M & $2,68 \%$ & 10 & 0 & 2 & 0 & 12 \\
\hline 10 & Schinus molle L. & Chorão & 34 & M & $2,07 \%$ & 2 & 2 & 6 & 3 & 13 \\
\hline 11 & $\begin{array}{l}\text { Lagerstroemia speciosa } \\
\text { Pers. }\end{array}$ & $\begin{array}{l}\text { Escumilha- } \\
\text { africana }\end{array}$ & 30 & M & $1,83 \%$ & 3 & 0 & 20 & 1 & 24 \\
\hline 12 & $\begin{array}{l}\text { Tecoma stans (L.) Juss } \\
\text { ex Kunth }\end{array}$ & Ipê-mirim & 28 & $\mathrm{M}$ & $1,70 \%$ & 6 & 1 & 0 & 0 & 7 \\
\hline 13 & Terminalia catappa L. & Sete-copas & 28 & G & $1,70 \%$ & 1 & 3 & 12 & 8 & 24 \\
\hline 14 & $\begin{array}{l}\text { Tibouchina granulosa } \\
\text { (Desr.) Cogn. }\end{array}$ & Quaresmeira & 27 & $\mathrm{G}$ & $1,64 \%$ & 1 & 3 & 8 & 1 & 13 \\
\hline 15 & $\begin{array}{l}\text { Jacaranda mimosifolia } \\
\text { D.Don. }\end{array}$ & $\begin{array}{l}\text { Jacarandá- } \\
\text { mimoso }\end{array}$ & 25 & $\mathrm{G}$ & $1,52 \%$ & 0 & 2 & 1 & 0 & 3 \\
\hline 16 & $\begin{array}{l}\text { Leucaena leucocephala } \\
\text { (Lam.) R. de Wit }\end{array}$ & Leucena & 25 & M & $1,52 \%$ & 0 & 2 & 7 & 1 & 10 \\
\hline 17 & $\begin{array}{l}\text { Handroanthus } \\
\text { heptaphyllus (Vell.) } \\
\text { Toledo }\end{array}$ & $\begin{array}{l}\text { Ipê-roxo-de- } \\
\text { sete-folhas }\end{array}$ & 24 & $G$ & $1,46 \%$ & 3 & 3 & 0 & 2 & 8 \\
\hline 18 & $\begin{array}{l}\text { Lafoensia glyptocarpa } \\
\text { Koehne }\end{array}$ & Mirindiba & 23 & $\mathrm{G}$ & $1,40 \%$ & 3 & 5 & 4 & 8 & 20 \\
\hline 19 & Michelia champaca L. & Magnólia & 21 & $M$ & $1,28 \%$ & 1 & 1 & 16 & 10 & 28 \\
\hline 20 & Mangifera indica L. & Mangueira & 20 & $\mathrm{G}$ & $1,22 \%$ & 1 & 4 & 3 & 0 & 8 \\
\hline- & Soma das $20+$ & & 1.242 & - & $75,59 \%$ & 81 & 112 & 276 & 238 & $\begin{array}{c}707 \\
(43,03 \%)\end{array}$ \\
\hline- & Soma Geral (1 a 130) & & 1.643 & - & $100,00 \%$ & 116 & 153 & 325 & 265 & $\begin{array}{c}859 \\
(52,28 \%)\end{array}$ \\
\hline
\end{tabular}

"Obs.: O item "Soma das 20+" (negrito), refere-se às análises quantitativas das modalidades de poda para as 20 espécies mais frequentes. O item "Soma Geral", refere-se às análises quantitativas das modalidades de poda para toda a população amostrada. 
Observou-se que a grande parte das espécies era de médio e grande porte, inadequados ao convívio com a rede elétrica, pois inevitavelmente têm potencial para provocar conflitos com a fiação, o que demanda alguma intervenção, seja pela prefeitura, ou pela concessionária de energia elétrica. Apenas três espécies da lista das 20 mais frequentes era de pequeno porte, Murraya paniculata, Hibiscus rosa-sinensis e Lagerstroemia indica, adequadas ao plantio sob rede elétrica sob a ótica do potencial para o conflito com a fiação.

Observou-se ainda, que 746 indivíduos apresentavam altura superior a seis metros, ou seja, 45,40\% da população, sendo 172 indivíduos de Poincianella pluviosa e 115 indivíduos de Licania tomentosa, o que é explicado pelos seus portes característicos, incompatíveis ao convívio com a rede elétrica.

Quanto às modalidades de poda executadas, verificou-se que a destopa foi a mais utilizada (325 indivíduos no total e 276 indivíduos para as 20 espécies mais frequentes), seguida da poda "em V" (265 indivíduos no total e 238 indivíduos para as 20 espécies mais frequentes), unilateral (153 indivíduos no total e 112 indivíduos para as 20 espécies mais frequentes) e, condução ou formação (116 indivíduos no total e 81 indivíduos para as 20 espécies mais frequentes).

Ressalta-se que a modalidade de poda "destopa" para a espécie Licania tomentosa foi uma prática perceptível nas regionais Leste, Norte e Triângulo, onde os espécimes apresentaram conformações estéticas com o objetivo principal de fornecimento de sombra.

Em uma análise mais aprofundada, observou-se que as cinco espécies com maior quantitativo de indivíduos podados por causa da rede elétrica nas quatro modalidades, perfizeram 59,48\% da população, sendo espécies de médio e grande porte, com potencial para gerar conflitos com a rede elétrica. Essas espécies foram: Licania tomentosa (202 indivíduos - 23,51\% - grande porte), Poincianella pluviosa (187 indivíduos - 21,76\% - grande porte), Ligustrum lucidum (55 indivíduos - 6,40\% - médio porte), Ficus benjamina (35 indivíduos - 4,07\% - grande porte) e Bauhinia variegata (32 indivíduos - 3,72\% - médio porte).

Por meio da análise da quantidade de indivíduos podados com causa rede elétrica (859), verificou-se que as 20 espécies mais frequentes corresponderam a $82,30 \%$ desse total, o que justificaria a elaboração de um plano de manejo de poda de árvores entre prefeituras e concessionária de energia elétrica. Esse plano de manejo seria bastante eficaz, uma vez que teriam prioridade de manutenção as espécies mais frequentes nas cidades, e que correspondem ao maior efetivo de podas realizadas com a causa rede elétrica, atingindo boa parte da população. 
A adoção de ciclos de podas para as espécies mais frequentes em uma dada população seria uma boa estratégia a ser incorporada pela concessionária, visto que reduziria grande parte dos desligamentos de energia elétrica, diminuiria os gastos com a manutenção da rede relativa aos galhos conflituosos podados com frequência, além de que manteria os espécimes mais saudáveis, inclusive esteticamente. Além disso, os resíduos de poda poderiam ser aproveitados pelas empresas para a geração de energia renovável, dado ao grande volume de madeira gerado todos os anos, pois, segundo Velázquez-Martí et al. (2014), tais resíduos contêm quantidades significativas de biomassa lignino-celulósicas, ou seja, características energéticas consideráveis.

Realizou-se a análise de agrupamento UPGMA (Figura 1), a partir da matriz de quantidade de indivíduos das espécies mais frequentes, relacionada às modalidades de podas executadas por causa da rede elétrica. O dendrograma com maior coeficiente de correlação $(0,9939)$ foi obtido por meio do índice de dados de abundância baseado na distância Euclidiana.

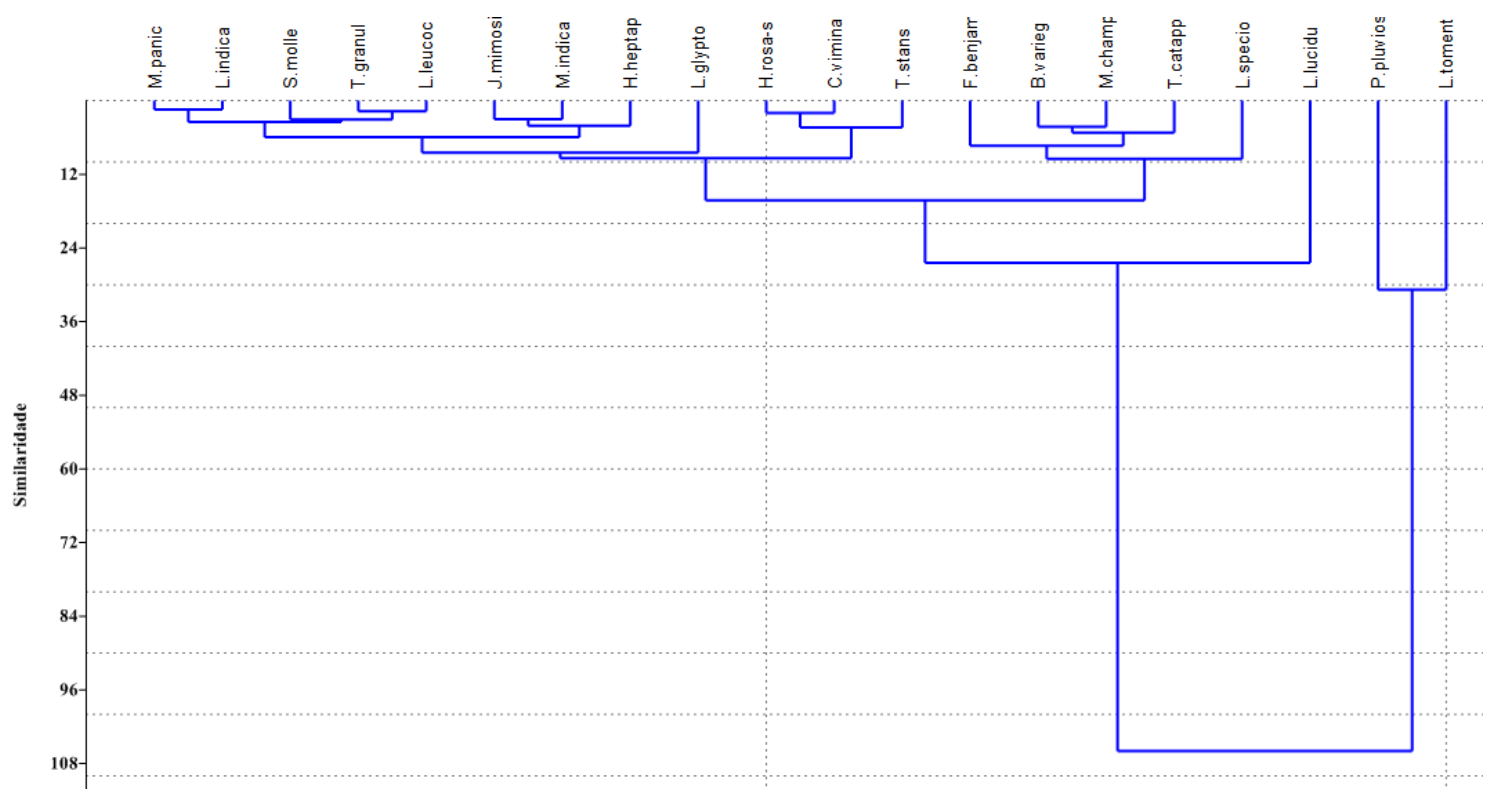

Figura 1 Dendrograma resultante da análise de agrupamento baseada na quantidade de indivíduos podados das 20 espécies mais frequentes encontradas no diagnóstico da arborização viária sob rede elétrica no Estado de Minas Gerais, relacionadas às quatro modalidades de poda (Condução ou Formação, Unilateral, Destopa e "em V", Tabela 1). O agrupamento foi realizado com base no método UPGMA, de distância Euclidiana. Coeficiente de correlação $=0,9939$

Figure 1 Resulting dendrogram from the cluster analysis based on the pruned trees amount for the 20 most frequent species found in the diagnosis of the trees that were located under power grids in the State of Minas Gerais-Brazil, related to the four types of pruning (Forming or Conducting, Unilateral, Topping and "V", Table 1). The cluster were performed based in the UPGMA method, Euclidian distance. Correlation coefficient $=0.9939$ 
Verificou-se a formação de dois grupos principais, separando Licania tomentosa e Poincianella pluviosa (primeiro grupo, direita), das demais espécies (segundo grupo, esquerda). Essas duas espécies apresentaram os maiores percentuais de poda dentro das quatro modalidades por causa da rede elétrica, motivo pelo qual se dispuseram em um grupo à parte.

No segundo grupo houve a formação de dois sub-grupos, sendo um deles composto apenas por Ligustrum lucidum, a qual foi a terceira espécie mais frequente dentro das modalidades de poda por causa da rede elétrica.

A formação de dois outros sub-grupos mostrou a divisão nas espécies que tiveram o quantitativo médio de podas (Ficus benjamina, Bauhinia variegata, Michelia champaca, Terminalia catappa e Lagerstroemia speciosa) daquelas que menos sofreram podas (Murraya paniculata, Lagerstroemia indica, Schinus molle, Tibouchina granulosa, Leucaena leucocephala, Jacaranda mimosifolia, Mangifera indica, Handroanthus heptaphyllus, Lafoensia glyptocarpa, Hibiscus rosa-sinensis, Callistemon viminalis e Tecoma stans).

Conclui-se assim, que as espécies de médio e grande porte, e que, consequentemente apresentaram os maiores quantitativos de espécimes podados, foram distribuídas nos grupos e subgrupos mais à direita do dendrograma, correspondendo às espécies mais problemáticas em se falando de compatibilidade com fiações elétricas.

A metodologia em questão pode ser utilizada para análises de urgência de adequação das espécies mais problemáticas, em se falando de modalidades de podas e porte de árvores. Com os resultados de análises amostrais da situação fitossanitária das espécies, essa análise pode se mostrar ainda mais profícua, gerando resultados bastante enriquecedores às instituições que lidam com o manejo da arborização viária.

A análise de correspondência canônica (Figura 2) objetivou estudar a relação entre a distribuição das 20 espécies mais frequentes no diagnóstico e a quantidade de indivíduos que sofreram intervenções de poda para essas espécies, com relação à rede elétrica.

As modalidades de poda distribuíram-se em duas dimensões, explicando 90,4\% da distribuição das espécies (Eixo/Dimensão 1=64,49\% e Eixo/Dimensão 2=25,91\%).

Ao longo do Eixo 1, eixo principal, a modalidade de poda "Condução ou Formação" ficou localizada na faixa da direita, em que se associaram Hibiscus rosa-sinensis, Tecoma stans e Calistemon viminallis, pois apresentaram a grande maioria dos seus indivíduos podados nessa modalidade. Isso pode ser em função das características de pequeno e médio porte dessas espécies, com exigência de menores intensidades de poda. 


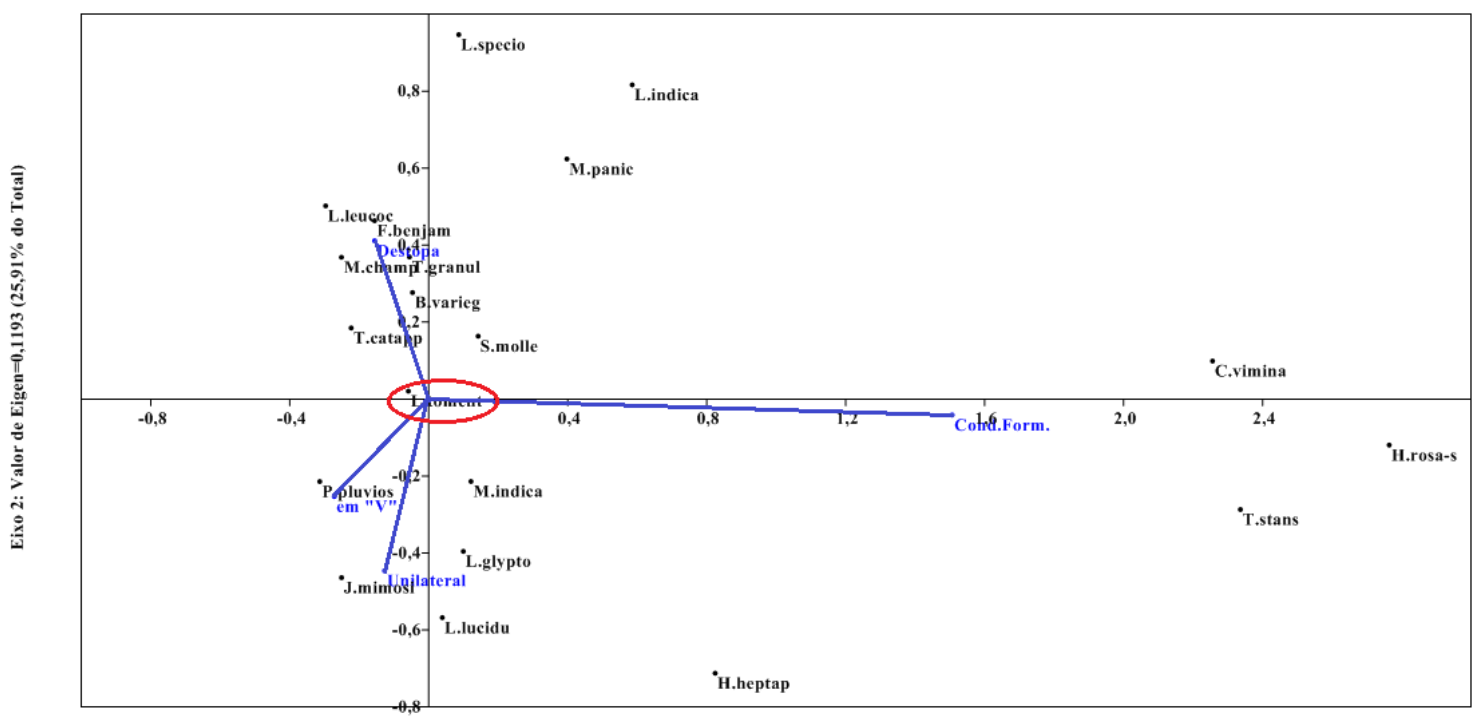

Eixo 1: Valor de Eigen $=0,2969(64,49 \%$ do Total)

Figura 2 Diagrama da análise de correspondência canônica (CCA) baseada nas 20 espécies mais frequentes encontradas no diagnóstico da arborização viária sob rede elétrica no Estado de Minas Gerais, e as quatro modalidades de poda relacionadas à rede elétrica (Condução ou Formação, Unilateral, Destopa e "em V", Tabela 30). Eixo 1: Valor de Eigen=0,2969 $(64,49 \%$ do total). Eixo 2: Valor de Eigen=0,1193 (25,91\% do total). Eixo 3: Valor de Eigen $=0,044$ (9,58\% do total)

Figure 2 Canonical correspondence analysis (CCA) diagram based in the 20 most frequent species found in the diagnosis of the trees that were located under power grids in the State of Minas Gerais-Brazil, and four pruning types related to the power grids (Forming or Conducting, Unilateral, Topping and "V", Table 1). Axis 1: Eigen value $=0.2969$ ( $64.49 \%$ of total). Axis 2: Eigen value $=0.1193(25.91 \%$ of total). Axis 3: Eigen value $=0.044$ (9.58\% of total)

$\mathrm{Na}$ faixa da esquerda se dispuseram as modalidades de poda "Destopa", "em V" e "Unilateral". O grupo composto por Leucaena leucocephala, Ficus benjamina, Michelia champaca, Tibouchina granulosa, Bauhinia variegata, Terminalia catappa e Licania tomentosa, se destacou sendo responsivo à modalidade "Destopa". Ao verificar a quantidade de todos os indivíduos destopados no diagnóstico, percebeu-se que essas espécies corresponderam a quase metade $(49,84 \%)$ desse montante, sendo a maioria de grande porte, incompatíveis com a fiação elétrica. Segundo estudo realizado por Kuhns e Reiter (2007), em seis cidades da porção oeste dos Estados Unidos, podas drásticas como a destopa, são percebidas de modo negativo pelos clientes das concessionárias de energia elétrica, principalmente devido à aparência estética desagradável em que as árvores se apresentam após as intervenções.

Analisando a faixa inferior do Eixo 2, houve destaque para Poincianella pluviosa, responsiva à modalidade de poda "em V" devido à grande quantidade de indivíduos que sofreram essa interferência. Há que se considerar o grande porte da espécie (de origem nativa) e o uso massivo em todo o território brasileiro, no qual atestou sua adaptação, por meio de plantios longevos em vários ecossistemas (BOBROWSKI, 2011). Apesar de essa 
espécie apresentar arquitetura que permite a realização de podas "em V", critérios devem ser levados em consideração para o plantio nos espaços urbanos, principalmente sob redes elétricas.

Por fim, sendo a espécie que apresentou maior quantidade de indivíduos no somatório de todas as modalidades de poda realizadas no diagnóstico, a Licania tomentosa se apresentou na porção central dos eixos, confirmando seu destaque.

Conforme estudos realizados em túneis verdes, ou seja, árvores podadas formando túneis para permitir a presença de redes aéreas, na cidade de Porto Alegre-RS, a utilização de espécies de grande porte em projetos de arborização fazem com que as concessionárias demandem muitos serviços de poda para manter a compatibilização entre as árvores e as redes de energia elétrica, e uma das soluções para podas excessivas seria a substituição de redes nuas por redes protegidas ou isoladas, já que demandam menor quantidade de podas e uma intervenção menos agressiva (SALVI et al., 2011).

De acordo com Loboda e De Angelis (2005), espécies de grande porte e espécies de crescimento monopodial são visivelmente mais problemáticas em se falando de compatibilização com rede elétrica e de telefonia. Em estudo realizado na cidade de Guarapuava-PR, os autores indicaram o plantio de espécies de pequeno porte sob rede e em locais onde o passeio é estreito, sugerindo que a concessionária de energia elétrica e a prefeitura municipal tenham plano integrado para arborização e sistemas elétricos. Estudos avançados em Sistemas de Informações Geográficas (SIG) são tendências que podem auxiliar a gestão da floresta urbana, pois trazem ferramentas capazes de mapear com precisão as espécies florestais na urbe, o que é um passo importante para o entendimento do contexto em que se encontram as florestas urbanas (ALONZO; BOOKHAGEN; ROBERTS, 2014).

\section{CONCLUSÕES}

Pouco mais da metade das árvores avaliadas (52,28\%) apresentaram poda motivada por conflitos com a fiação elétrica, sendo que o percentual de 43,03\% das árvores era relativo às 20 espécies mais frequentes, o que reflete a considerável quantidade de indivíduos com altura superior a seis metros avaliados no diagnóstico total (45,40\%). As três espécies mais podadas foram Licania tomentosa, Poincianella pluviosa e Ligustrum lucidum, e a modalidade de poda "destopa" foi a mais frequente, seguida da poda "em V", adotada pelas concessionárias de energia elétrica, com o objetivo de compatibilização entre árvores e redes elétricas. 
Com base em tais resultados sugerem-se maiores estudos quanto ao ciclo de poda para as espécies que atualmente estão em conflito com a rede de distribuição de energia elétrica. Caso as referidas espécies mais frequentes sejam melhor avaliadas, em um processo sistemático e prioritário (inclusive com o auxílio de SIGs), conseguir-se-ia um quantitativo bastante significativo de intervenções, que iria contribuir efetivamente para a diminuição das ocorrências de desligamento em virtude de conflitos com árvores. Além disso, esse estudo possibilitaria melhor adaptação a espécies que não são tão afins a sofrer sucessivas podas.

\section{AGRADECIMENTOS}

À Gerência de Gestão Ambiental da Cemig Distribuição S.A., por meio do Programa de Pesquisa e Desenvolvimento, PeD-D428, parceria entre Cemig e Agência Nacional de Energia Elétrica (Aneel) e Universidade Federal de Lavras (Ufla).

\section{REFERÊNCIAS}

AGUIRRE JUNIOR, J. H.; LIMA, A. M. L. P. Uso de árvores e arbustos em cidades brasileiras. Revista da Sociedade Brasileira de Arborização Urbana, Piracicaba, v. 2, n. 4, p. 50-66, 2007.

ALONZO, M.; BOOKHAGEN, B.; ROBERTS, D.A. Urban tree species mapping using hyperspectral and lidar data fusion. Remote Sensing of Environment, Minnesota, v. 148, p. 70-83, 2014.

APPLETON, B.L. Designing and implementing utility line arboreta. Arboriculture \& Urban Forestry, Champaign, v.32, n.2, mar., 2006.

ARAUJO, A. C. B.; GRACIOLI, C. R. G.; GRIMM, E. L.; LONGHI, S. J. Avaliação da florística, do porte e da fitossanidade atual da arborização do Parque Internacional em Sant'ana do Livramento/Rivera, Brasil/Uruguai. Revista da Sociedade Brasileira de Arborização Urbana, Piracicaba, v. 7, n. 1, p. 112-125, 2012.

BOBROWSKI, R. Estrutura e dinâmica da arborização de ruas de Curitiba-Paraná, no período 1984-2010. 144f. Dissertação (Mestrado em Engenharia Florestal), Programa de Pós-graduação em Engenharia Florestal - Universidade Federal do Paraná, Curitiba, PR, 2011.

CAMPANELLA, B.; TOUSSAINT, A.; PAUL, R. Mid-term economical consequences of roadside tree topping. Urban Forestry \& Urban Greening, Davis, v. 8, p. 49-53, 2009.

COMPANHIA ENERGÉTICA DE MINAS GERAIS. Manual de arborização. Belo HorizonteMG: Cemig/Fundação Biodiversitas, 2011. 111p. 
COMPANHIA ENERGÉTICA DE MINAS GERAIS. Manual de arborização. Belo Horizonte: Superintendência de Comunicação Social e Representação, 1996. 40 p.

COMPANHIA ENERGÉTICA DE MINAS GERAIS. Manual de distribuição: norma de distribuição-ND-2.1: instalações básicas de redes de distribuição aéreas urbanas. Belo Horizonte, 2002. $186 \mathrm{p}$.

CUPERTINO, M. A.; EISENLOHR, P. V. Análise florística comparativa da arborização urbana nos campi universitários do Brasil. Bioscience Journal, Uberlândia, v. 29, n. 3, p. 739-750, 2013.

HAMMER, Ø; HARPER, D. A. T.; RYAN, P. D. Past: Paleontological Statistics Software Package for Education and Data Analysis. Palaeontologia Electronica, Columbia, v. 4, n. 1, p. 01-09, 2001.

KUHNS, M.R.; REITER, D.K. Knowledge of and attitudes about utility pruning and how education can help. Arboriculture \& Urban Forestry, Champaign, v.33, n.4, p.264-274, 2007.

LISTA DE ESPÉCIES DA FLORA DO BRASIL. Disponível em: <http://floradobrasil.jbrj. gov.br/2012>. Acesso em: 9 set. 2013.

LOBODA, C.R., DE ANGELIS, B.L.D., Áreas verdes públicas urbanas: conceitos, usos e funções. Revista Ambiência, Guarapuava, v.1, n.1, Jan./Jun., 2005.

MELAZO, G. C.; NISHIYAMA, L. Mapeamento da cobertura arbóreo-arbustiva em quatro bairros da cidade de Uberlândia, MG. Revista da Sociedade Brasileira de Arborização Urbana, Piracicaba, v. 5, n. 2, p. 52-66, 2010.

MULLANEY, J.; LUCKE, T.; TRUEMAN, S.J. A review of benefits and challenges in growing street trees in paved urban environments. Landscape and Urban Planning, Amsterdam, v. 134, p.157-166, 2015.

NOWAK, D. J.; MCBRIDE, J. R.; BEATTY, R. A. Newly planted street tree growth and mortality. Journal of Arboriculture, Champaign, v. 16, n. 05, p.124-129. 1990.

ROY, S; BYRNE, J.; PICKERING, C. A systematic quantitative review of urban tree benefits, costs, and assessment methods across cities in different climatic zones. Urban Forestry \& Urban Greening, Davis, v. 11, p.351-363, 2012.

SALVI, L.T.; HARDT, L.P.A.; ROVEDDER, C.E.; FONTANA, C.S. Arborização ao longo de ruas - túneis verdes - Porto Alegre, RS, Brasil: avaliação quantitativa e qualitativa. Revista Árvore, Viçosa, v.35, n.2, p.233-243, 2011.

THE PLANT LIST-a working list of all plant species. Disponível em: <http://www.theplant list.org/>. Acesso em: 9 set. 2013.

VELÁZQUEZ-MARTÍ, B; SAJDAK, M.; LÓPEZ-CORTÉS, I.; CALLEJÓN-FERRE, A.J. Wood characterization for energy application proceeding from pruning Morus alba L., Platanus hispanica Münchh. and Sophora japonica L. in urban areas. Renewable Energy, Cyprus, v. 62, p.478-483, 2014. 